\title{
ALIMENTAÇÃO DE UM FILHOTE DE BEM-TE-VI, PITANGUS SULPHURATUS (LINNAEUS) \\ (PASSERIFORMES, TYRANNIDAE), EM AMBIENTE URBANO
}

\author{
Maria Martha Argel-de-Oliveira ${ }^{1,2}$ \\ Nancy A. Curi ${ }^{1}$ \\ Tatiana Passerini ${ }^{1}$
}

\begin{abstract}
FeEding of a fledgling great kiskadeE, Pitangus sulphuratus (LINNAEUS) (PASSERIFORMES, TYRANNIDAE) IN URBAN ENVIRONMENT. The observation of parents feeding a captive fledgling of Great Kiskadee revealed that visitation is more frequent in the first hour after sunrising, with lesser peaks along the day. The diet supplied consisted mainly of insects (Hymenoptera, Lepidoptera, Coleoptera, Diptera), along with other items as human-made materials (food scraps, pellets of animal food), lizards (Hemidactylus mabouia), non-identified fruit pulp, gastropods and arachnids (Opiliones). The ability that $P$. sulphuratus has for identifying food items absent from more natural habitats and for exploiting resources of unpredictable spatial and temporal distributions confers the species a dietary flexibility that probably contributes to its efficiency in colonizing urban habitats.

KEY WORDS. Passeriformes, Tyrannidae, Pitangus sulphuratus, diet, urban environment
\end{abstract}

As inúmeras aves nativas que conseguem sobreviver e se estabelecer em ambiente urbano enfrentam o desafio de explorar recursos que são diferentes ou que estão presentes em quantidades diferentes das que ocorrem em ambientes naturais.

A composição da avifauna tem sido estudada em várias cidades brasileiras, mas pouco se sabe sobre a biologia das aves no ambiente urbano, dominado por elementos criados pelo ser humano, e sobre a forma como elas exploram os recursos disponiveis. Alguns trabalhos existem (HILL \& SCHERER-NeTO 1991; ONIKI et al. 1992; RUSZCZYK et al. 1987; SICK \& TEIXEIRA 1981; SILVA 1988), mas são isolados e não detalhados.

O bem-te-vi Pitangus sulphuratus (Linnaeus, 1766) (Tyrannidae) é uma das aves mais comuns nas cidades de todo o Brasil, sendo abundante em metrópoles como São Paulo (ARGEL-DE-OliveIRA 1995), Rio de Janeiro (SICK 1997) e Porto Alegre (RUSZCZYK et al. 1987). Não existem estudos sobre aspectos de sua biologia no ambiente urbano, como por exemplo a composição da dieta. Sobre este assunto, mesmo em ambientes menos antropizados são escassos os estudos (BELTZER 1983), em que pese a ampla distribuição geográfica da espécie e as inúmeras citações

1) Faculdades Integradas São Camilo. Avenida Nazaré 1501, 04263-200 São Paulo, São Paulo, Brasil.

2) Endereço para correspondência: Avenida Irerê 198, 04064-000 São Paulo, São Paulo, Brasil.

E-mail: tita@originet.com.br 
isoladas a sua dieta (CARVALHO 1960; FFRENCH 1980; HAVERSCHMIDT 1968; SICK 1997).

O objetivo deste trabalho foi obter informações quanto à alimentação de um jovem de $P$. sulphuratus em uma situação urbana. Basicamente procurou-se saber com que freqüência o alimento era fornecido pelos pais e qual a composição da dieta oferecida.

\section{MATERIAL E MÉTODOS}

O estudo foi desenvolvido em São Paulo, São Paulo, Brasil, em um bairro residencial arborizado. O local das observações dista menos de $50 \mathrm{~m}$ do pátio de uma escola, freqüentado pelos alunos nos intervalos entre as aulas. Uma descrição mais detalhada da área consta de ARGEL-DE-OLIVEIRA (1995).

Em 27 de novembro de 1994, um filhote já capaz de curtos vôos foi recolhido na rua, encharcado após chuvas fortes. Entre 28 a 30 de novembro foi mantido em gaiola, longe de movimentação humana e a menos de $10 \mathrm{~m}$ do local onde foi recolhido, de forma que a visitação por adultos era possível. A movimentação dos adultos e o fornecimento de alimento foram acompanhados por $29 \mathrm{~h} 10 \mathrm{~min}$, que abrangiam todo o período desde a primeira luz até o escurecer, cobrindo todo o período de atividade das aves. As observações foram feitas com binóculos (ZeissJena $8 \times 32$ ), desde uma distância de $6 \mathrm{~m}$, através de uma vidraça. A presença do observador aparentemente passou despercebida às aves e parece não ter constituído uma fonte de perturbação a seu comportamento. Foi anotada a freqüência de visitação pelos pais e, quando possível, identificados visualmente os itens trazidos. Itens não ingeridos pelo filhote, derrubados no fundo da gaiola, eram removidos assim que o(s) adulto(s) se afastava(m), identificados, medidos com paquímetro e colocados fora da gaiola. Isso era feito porque muitas vezes as grades da gaiola atrapalhavam a transferência de alimento. Alimentos caídos eram ignorados pelo filhote; quando colocados do lado de fora, eram recolhidos e reapresentados pelos adultos.

Durante as 50 horas (incluindo o período noturno) em que o filhote foi mantido na gaiola para estudo, foram recolhidos 79 fezes e 10 regurgitados. Posteriormente, foram dissolvidos em álcool e triados em laboratório, sob microscópio estereoscópico, para confirmação da identidade das presas. A identificação do material foi feita até o menor nível taxonômico possível, que no caso de insetos era, em geral, a Ordem.

\section{RESULTADOS}

\section{Visitação}

Ao todo, foram registradas 153 visitas. Havia mais de um adulto envolvido na atividade de alimentação, pois em três ocasiões houve visitas simultâneas feitas por duas aves, provavelmente os pais. À aproximação de um adulto, o filhote pedincha: acerca-se às grades da gaiola, com o bico aberto e tremulando as asas, enquanto emite uma vocalização insistente, típica dos filhotes e sem semelhança com as notas do adulto. 
O intervalo médio entre visitas foi de $10,4 \pm 14,1 \mathrm{~min}(\mathrm{n}=147)$, tendo sido verificados desde visitas simultâneas até um lapso de 106 min separando duas visitas.

Como se vê na figura 1 , as visitas eram mais seguidas nas primeiras horas da manhã. Entre as 5:00 e as 8:00 horas, o intervalo médio entre visitas foi de 4,8 minutos, sendo que a visitação era conspicuamente mais freqüente logo na primeira hora da manhã, antes das 6:00 horas, o intervalo médio entre visitas foi de 2,2 minutos. No segundo e no terceiro dias de observação, a intensa atividade das primeiras horas da manhã correspondeu a um fornecimento intenso de formigas aladas, um recurso abundante, já que estavam ocorrendo revoadas desse inseto.

Os intervalos mais longos foram registrados durante as horas mais quentes do dia: entre as 12:00 horas e as 15:00 horas, a média foi de 25,4 min. O intervalo mais longo ocorreu entre as 13:00 e as 15:00 horas, após o filhote aceitar e engolir inteira uma lagartixa, Hemidactylus mabouia (Moreau de Jonnès, 1818) (Gekkonidae): a visita seguinte ocorreu depois de 106 minutos; nessa, e nas três visitas seguintes, o filhote permaneceu em silêncio e fez poucas tentativas de alcançar os alimentos oferecidos (um deles, outra lagartixa). O adulto afastava-se com o alimento ou o ingeria no local. O filhote só voltou a aceitar alimento $202 \mathrm{~min}$ após a ingestão da lagartixa, retomando o comportamento de pedinchar. A partir daí, regularizou-se a visitação, aumentando sua freqüência.

\section{Composição da dieta}

Foram oferecidas ao filhote presas animais (insetos, pequenos répteis, gastrópodos e um opilião), vegetais (polpa de fruto) e substâncias manufaturadas pelo ser humano, como restos de alimentos humanos cuja base é o amido, pellets de rações animais e até mesmo itens não alimentares, como barbante.

A relação dos itens identificados é apresentada na tabela I. Isoladamente, nem a observação do comportamento de visitação nem a análise de fezes e de regurgitados teriam sido suficientes para revelar todo o espectro que a combinação dos dois métodos revelou.

Das 96 visitas em que foi identificado o tipo de alimento trazido pelos adultos, $64 \%$ corresponderam a presas animais $(n=61)$, sendo as presas mais numerosas as formigas aladas $(n=40)$; polpa de fruto foi trazida em uma única visita ( $1 \%$ ); alimentos manufaturados pelo ser humano foram fornecidos em 32 visitas $(33 \%)$ e substâncias que na verdade não serviam como alimento foram trazidas em duas ocasiões ( $2 \%)$.

Como já foi mencionado, as formigas aladas foram abundantes durante parte do período de estudo. Restos de alimentos industrializados provavelmente eram recolhidos no pátio da escola.

Nem todos os itens eram ingeridos pelo filhote. $\mathrm{O}$ opilião foi recusado, o mesmo ocorrendo muitas vezes com os salgadinhos. Apenas uma lagartixa foi ingerida - em duas visitas imediatamente posteriores foram oferecidas uma cauda e outra lagartixa, mas o filhote não manifestou interesse. Em geral os adultos ingeriam os itens recusados, incluindo formigas, bolo, pão e a lagartixa. 


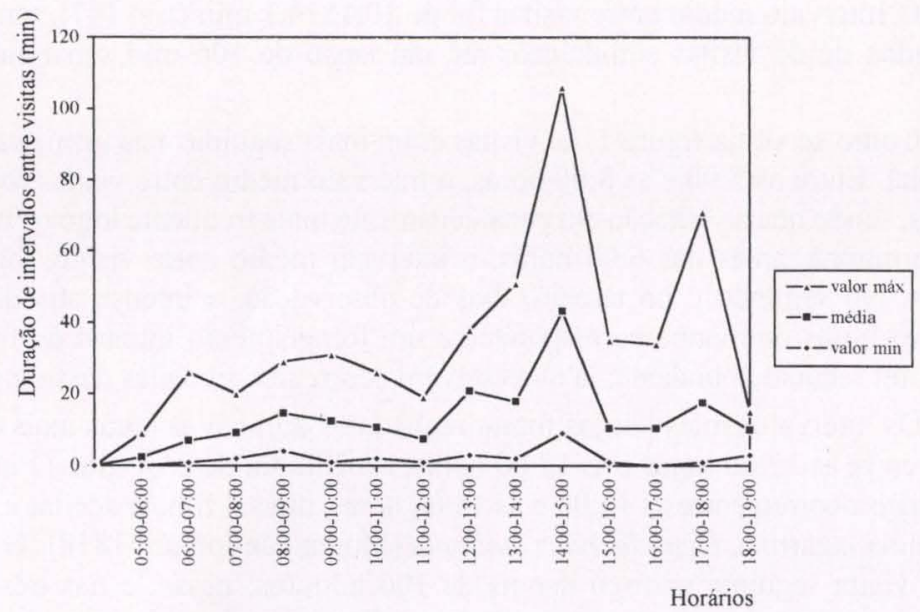

Fig. 1. Duração dos intervalos entre visitas feitas por adultos de Pitangus sulphuratus a um jovem, de acordo com o horário. Foi feito um pool com os dados registrados ao longo dos três dias de estudo (28-30-XI-1994).

Tabela I. Itens alimentares identificados através de observação do comportamento de visitação de adultos e da análise de fezes e regurgitados de um filhote de Pitangus sulphuratus.

\begin{tabular}{|c|c|c|}
\hline Itens & & $\begin{array}{l}\text { tas } \\
\text { cido }\end{array}$ \\
\hline Mollusca Gastropoda & - & Fezes \\
\hline Arachnida Opiliones & 1 & - \\
\hline Coleoptera & 1 & Fezes e regurgitados \\
\hline Diptera & - & Fezes \\
\hline Lepidoptera & 12 & Fezes e regurgitados \\
\hline Hymenoptera (Formicidae) & 40 & Fezes e regurgitados \\
\hline Hymenoptera (outros) & - & Fezes e regurgitados \\
\hline Pupa de inseto & - & Regurgitados \\
\hline Insetos năo identificados & 4 & Fezes e regurgitados \\
\hline Reptilia (Hemydactilus mabouia) & 3 & Regurgitados \\
\hline \multicolumn{3}{|l|}{ Alimento de origem vegetal } \\
\hline Polpa de fruto & 1 & - \\
\hline \multicolumn{3}{|c|}{ Alimentos manufaturados pelo ser humano } \\
\hline Salgadinho & 12 & Fezes \\
\hline Păo & 8 & $?$ \\
\hline Bolo & 3 & $?$ \\
\hline Batata frita & 4 & $?$ \\
\hline Macarrão & 1 & $?$ \\
\hline Ração & 4 & Fezes \\
\hline \multicolumn{3}{|l|}{ Não alimento } \\
\hline Barbante & 2 & Regurgitados \\
\hline Itens não determinados & 57 & \\
\hline $\begin{array}{l}\text { Total visitas } \\
\text { Total de amostras analisadas }\end{array}$ & 153 & \\
\hline
\end{tabular}


As presas identificadas através da análise de fezes e regurgitados não foram quantificadas numericamente. Restos de insetos foram encontrados em todas as amostras à exceção de uma. O volume do material presente foi estimado para 77 amostras: em $70 \%$ dos casos o predomínio foi de restos de insetos; em $26 \%$ foi de materiais não determinados, provavelmente restos de produtos industrializados; em $4 \%$ foi do que pareciam ser restos de matéria vegetal. A maioria dos fragmentos encontrados em fezes era irreconhecível, e muitas das fezes se compunham quase exclusivamente por escamas de mariposas. Embora lepidópteros tenham sido oferecidos em apenas 12 ocasiões ( $8 \%$ de todas as visitas), seus vestígios apareceram em $70 \%$ das amostras, não só devido ao grande tamanho dessas presas mas também porque as escamas devem mesclar-se de tal forma ao conteúdo gástrico que sua completa eliminação é lenta. Partes reconhecíveis, como cabeças (incluindo de coleópteros e himenópteros), fragmentos maiores e mais quitinizados e ossos de $H$. mabouia apareceram principalmente nos regurgitados. Ossos provenientes de uma única lagartixa apareceram em três regurgitados. Considerando-se que, após a ingestão dessa presa o filhote não ingeriu nada mais por 3,3 horas, e que nesse período deve ter havido a eliminação de 6 fezes ou regurgitados, a lagartixa está subrepresentada nas amostras. O mesmo deve acontecer com outros itens cuja identificação depende em grande parte da presença em regurgitados.

Em geral não foi possível estimar o tamanho dos itens alimentares - durante a observação, por dificuldade de visualização, e na triagem, pelo estado fragmentado da maioria das presas - mas aparentemente são mais numerosas as presas com menos de $1 \mathrm{~cm}$. Foram abundantes as cabeças de himenópteros e besouros que não devem superar esse tamanho; tais insetos pequenos devem representar boa parte da carga transportada nas visitas em que o alimento oferecido não pode ser determinado, em geral por estar contido totalmente no interior do bico. As presas maiores foram os dois exemplares de H. mabouia, (comprimento estimado de 6 e $8 \mathrm{~cm}$ ). Uma das mariposas media $4,7 \mathrm{~cm}$ de corpo.

\section{DISCUSSÃO}

Na dieta observada houve um predomínio nítido de insetos: constituíram o item oferecido em maior número de visitas, tiveram a maior freqüência de aparecimento nas fezes e regurgitados e predominaram volumetricamente na maior parte das amostras. Essa constatação corrobora a conclusão de um estudo quantitativo feito na Argentina, segundo o qual os insetos compõem a fração mais importante da dieta da espécie (BELTZER 1983), a despeito da infinidade de citações a outros itens na literatura.

Essas listas, exaustivas, contêm os mais variados itens alimentares explorados por $P$. sulphuratus. Embora não quantifiquem seu consumo, são uma forte indicação de grande flexibilidade alimentar, o que é corroborado no presente estudo.

Consideremos primeiro o consumo de alimentos industrializados. Por um lado, a aparência de itens como salgadinhos, batatas fritas e pão não corresponde à de itens animais ou vegetais corriqueiramente consumidos na natureza, e seu consumo implicaria em um processo de aprendizado baseado em tentativa-e-erro. 
Os erros ocorrem, e isso explicaria o oferecimento e a aceitação de material não nutritivo (barbante) em duas ocasiões. Por outro, a exploração desses recursos representa uma resposta oportunista da ave a uma situação espacialmente peculiar: a presença de uma área - o pátio da escola - onde eles são particularmente passíveis de ser encontrados.

Outro aspecto da amplitude comportamental de $P$. sulphuratus é exemplificado pela exploração maciça, em parte do tempo de estudo, de formigas aladas que revoavam - revoadas são situações temporárias onde as presas aparecem em concentrações elevadas. $P$. sulphuratus explora de preferência presas presentes em densidades altas (CRUMP \& VAIRA 1991), e neste caso respondeu a um fenômeno súbito, uma disponibilidade de presas acima do comum e provavelmente não previsível, passando a explorá-lo intensamente, outra mostra de flexibilidade comportamental.

Em suma, a considerável flexibilidade alimentar em $P$. sulphuratus deve advir da combinação das capacidades de descobrir por tentativa-e-erro novos tipos de alimento e de explorar recursos localmente disponiveis ou disponíveis de forma irregular e imprevisível ao longo do tempo.

A amplitude potencial da dieta sem dúvida é um dos fatores que torna esta ave eficiente em colonizar novos ambientes, mas é provável que a flexibilidade comportamental se estenda a outros aspectos de sua biologia. A grande capacidade que tem de se estabelecer em novos ambientes pode ser avaliada pelos fato de ser, dentre as aves silvestres comuns na cidade de Porto Alegre, a mais abundante no centro (RUSZCZYK et al. 1987). A biologia de P. sulphuratus no ambiente urbano merece ser mais estudada e comparada com seus hábitos em ambientes menos antropizados. Talvez essa linha de pesquisa ajude a compreender como a antropização da paisagem determina, através da modificação dos tipos de recursos disponíveis, a composição da avifauna urbana. Essa compreensão é indispensável se, por motivos éticos, estéticos e conservacionistas, quisermos traçar estratégias de manejo que tornem as cidades mais hospitaleiras a avifaunas mais ricas e mais abundantes.

AGRADECIMENTOS. Agradecemos a Nelson Bomtempi, coordenador do curso de Ciências Biológicas das FISC, pelo apoio logístico, e a Marcos V. A. Lapa, pelo auxílio na triagem do material.

\section{REFERÊNCIAS BIBLIOGRÁFICAS}

ARGEL-DE-OlIVEIRA, M.M. 1995. Aves e vegetação em um bairro residencial da cidade de São Paulo. Revta bras. Zool. 12 (1): 81-92.

Beltzer, A.H. 1983. Alimentación del "benteveo" (Pitangus sulphuratus) en el valle aluvial del rio Paraná Medio. (Passeriformes: Tyrannidae). Revta Asoc. Cienc. nat. Litoral 14: 47-52.

Carvalho, C. T. 1960. Comportamento de Myiozetetes similis e notas biológicas sobre espécies afins (Passeres, Tyrannidae). Pap. Avulsos Depto Zool., São Paulo, 14 (15): 121-132.

CRUMP, M.L. \& M. VAIRA. 1991. Vulnerability of Pleurodema borelli tadpoles to 
an avian predator: effect of body size and density. Herpetologica 47 (3): 316-321.

FFRENCH, R. 1980. A guide to the birds of Trinidad and Tobago. Newtown

Square, Harrowood Books, 470p.

HAVERSCHMIDT, F. 1968. Birds of Surinam. Edinburgh, Oliver \& Boyd, 445p.

HILL III, J.R. \& P. SCHERER-NETO. 1991. Black Vultures nesting on skyscrapers in

Southern Brazil. Jour. Field Ornithol. 62 (2): 173-176.

ONIKI, Y.; E.O. Willis \& M.M. Willis. 1992. Chaetura andrei (Apodiformes, Apodidae): aspects of nesting. Ornitol. neotrop. 3: 65-68.

RuszCZYK, A.; J.J.S. Rodrigues; T.M.T. ROBERTS; M.M.A. BENDATI; R.S. DEL

PINO; J.C.V. MARQUES \& M.T.Q. MELO. 1987. Distribution patterns of eight bird species in the urbanization gradient of Porto Alegre, Brazil. Cienc. Cult. 39 (1): 14-19.

SicK, H. 1997. Ornitologia brasileira. Rio de Janeiro, Nova Fronteira, 862p.

SICK, H. \& D.M. TEIXEIRA. 1981. Nocturnal activities of Brazilian hummingbirds and flycatchers at artificial ilumination. Auk, Lawrence, 98: 191-192.

SILVA, J.M.C. 1988. Atividades noturnas de forrageamento, sob iluminação artificial, de algumas aves amazônicas. Bolm Mus. par. Emílio Goeldi, sér. zool., Belém, 4 (1): 17-20.

Recebido em 08.VIII.1997; aceito em 03.XII.1998 